\title{
ADAPTATION OF INDUSTRIAL HYPERSPECTRAL LINE SCANNER FOR ARCHAEOLOGICAL APPLICATIONS
}

\author{
V. Miljkovićc ${ }^{a, *}$, D. Gajski ${ }^{\text {a }}$ \\ ${ }^{a}$ Faculty of Geodesy, Institute of Cartography and Photogrammetry, Kačićeva 26, Zagreb, Croatia - (vmiljkovic, dgajski)@ geof.hr
}

COMMISSION V, WG V/2

KEY WORDS: hyperspectral scanning, archaeometry, calibration, cultural heritage, spectral classification, visualisation

\begin{abstract}
:
The spectral characteristic of the visible light reflected from any of archaeological artefact is the result of the interaction of its surface illuminated by incident light. Every particular surface depends on what material it is made of and/or which layers put on it has its spectral signature. Recent archaeometry recognises this information as very valuable data to extend present documentation of artefacts and as a new source for scientific exploration. However, the problem is having an appropriate hyperspectral imaging system available and adopted for applications in archaeology. In this paper, we present the new construction of the hyperspectral imaging system, made of industrial hyperspectral line scanner ImSpector V9 and CCD-sensor PixelView. The hyperspectral line scanner is calibrated geometrically, and hyperspectral data are geocoded and converted to the hyperspectral cube. The system abilities are evaluated for various archaeological artefacts made of different materials. Our experience in applications, visualisations, and interpretations of collected hyperspectral data are explored and presented.
\end{abstract}

\section{INTRODUCTION}

Hyperspectral images are defined as being recorded simultaneously in many, narrow, contiguous bands to provide information on the major features of the spectral reflectance of a given object. The images can be visualised as a 3-dimensional dataset with two spatial and one spectral dimension and the data set is therefore often referred to as an image cube. Originally, raw hyperspectral data are combined in an image cube with spatial, temporal and spectral dimension, after the imaging characteristic of the hyperspectral sensor (mostly push-broom scanner), and they have to be transformed to geocoded hyperspectral cube for all further spatial analysis of hyperspectral data. There are several methods to transform raw hyperspectral data (raw cube) into geocoded one. Because of imaging geometry of the hyperspectral sensor (push-broom scanner), only the parametric geocoding methods can be applied directly.

Hyperspectral imaging is one of the most emerging archaeometric technology. It brings a completely new set of information about artefacts, a complement to all other till now documented by standard archaeometric procedures. Reflexiveness of the surface of archaeological artefact taken in many narrow spectral bands over a whole visible and near-IR spectrum is valuable information that characterises the surface on the way it is possible to recognise it among many others, for human sight the same, surfaces. That's why the spectral reflexiveness is taken as spectral "signature" (Miljković, Gajski 2011)

\section{DATA ACQUISITION SYSTEM}

\subsection{Overall description}

The data acquisition system used in this research consist of hyperspectral sensor and scanning mechanism adapted for close-range imaging. The hyperspectral sensor consists of imaging spectrometer ImSpector V9 and matrix CCD-sensor PIXELFLY. The scanning mechanism is constructed in the form of a stable metal frame with movable sensor carrier.

\subsection{Hyperspectral sensor}

The ImSpector V9 is imaging spectrograph component both for industrial machine vision and analytical applications and for scientific spectroscopic work. The imaging spectrograph is "an instrument capable of simultaneously measuring the optical spectrum components and the spatial location of an object surface" (ImSpector User manual). Technically it is not possible to record simultaneously spectral information together with the 2-dimensional position of measured spectrum on object's surface. This leads to the idea that one dimension of the detector constitutes a spatial line image and the other dimension measures the spectrum for each line pixel (Figure 1).

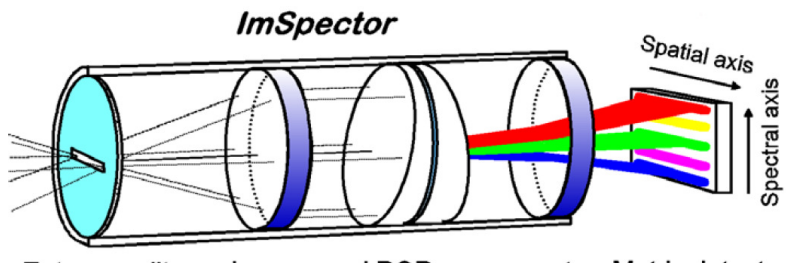

Entrance slit Lenses and PGP component Matrix detector

Figure 1. The principle of imaging spectrograph ImSpector (Lawrence et al.)

The spectral characteristics of ImSpector V9 spectrograph are: spectral range from 430-900nm and spectral resolution are $7 \mathrm{~nm}$ (with 80um slit)

The matrix detector we use in the hyperspectral sensor is PCO PIXELFLY HiRES (Figure 2, c). It is CCD-sensor with $1360 \times 1024$ photodetectors and 12-bit dynamic range. This sensor is mounted and oriented to ImSpector on the way that spatial axis corresponds to the direction of rows (with 1360pix per each). However, the spatial line has just 1180pix. The rest of 180pix is reserved for recording the intensity and spectrum of incident light. The part of the energy of incident radiation is taken by diffuse collector - Phodis (Figure 2, b) and by optical 
cable led to Imspector V9 (Figure 2, d) This information is necessary to calculate the reflectivity of object's surface.

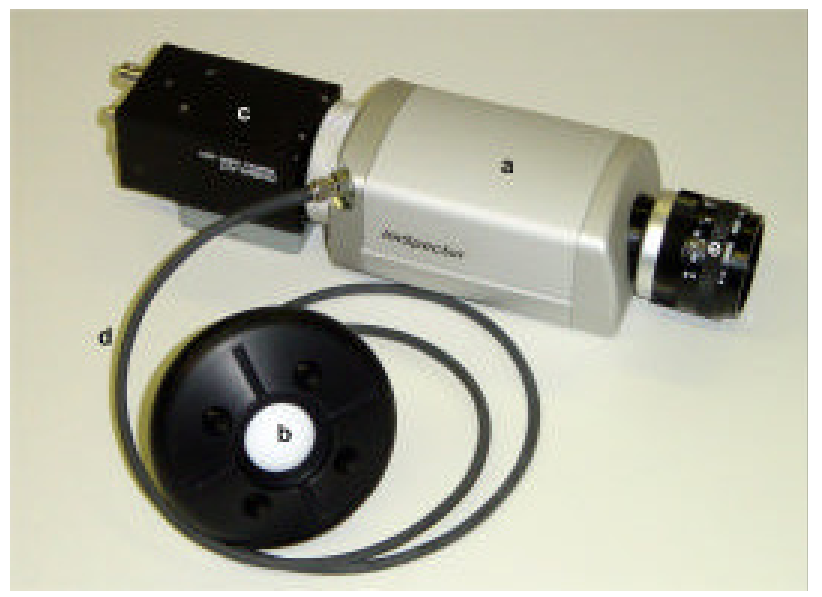

Figure 2. Imaging Spectrograph ImSpector V9 (a); diffuse collector of incident light - Phodis (b); CCD-sensor PCO PixelFly; Optic cable (d); Objective lens Schneider-Kreuznach $\mathrm{f}=24 \mathrm{~mm}(\mathrm{e})$ (Bajić et al., 2004)

\subsection{Scanning mechanism}

The stable metal frame holds the movable carrier of the hyperspectral sensor (Figure 3, a+b) and illumination source (Figure 3, d). The illumination source is moving together with the hyperspectral sensor, ensuring that the samples are always optimally illuminated, almost without shadows. The chosen illumination source is a halogen lamp, because of its smooth spectral characteristics, without significant peaks over a whole visible and NIR spectrum.

The diffuse collector of incident light (Figure 3, c) collects the light spectrum directly from the lamp. The computer controls the start and end position of the hyperspectral sensor on the scanner, as well as the velocity during scanning.

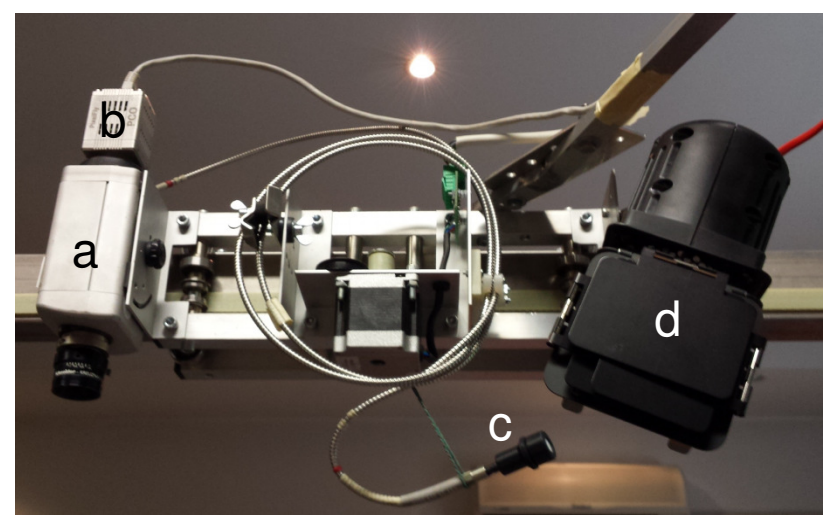

Figure 3. Movable carrier of scanner with ImSpector V9 (a), CCD sensor PixelFly (b), diffuse collector of incident light (c) and source of light $(\mathrm{d})$

\section{CASE STUDY}

The described hyperspectral scanner is suitable to collect spectral data, taken from fragments of archaeological artefacts with the goal to find the correlation among those, originating from the same artefact.

\subsection{Description of chosen fragments}

To simulate the real situation, we use the set of real archaeological fragments, as shown in figure 4.

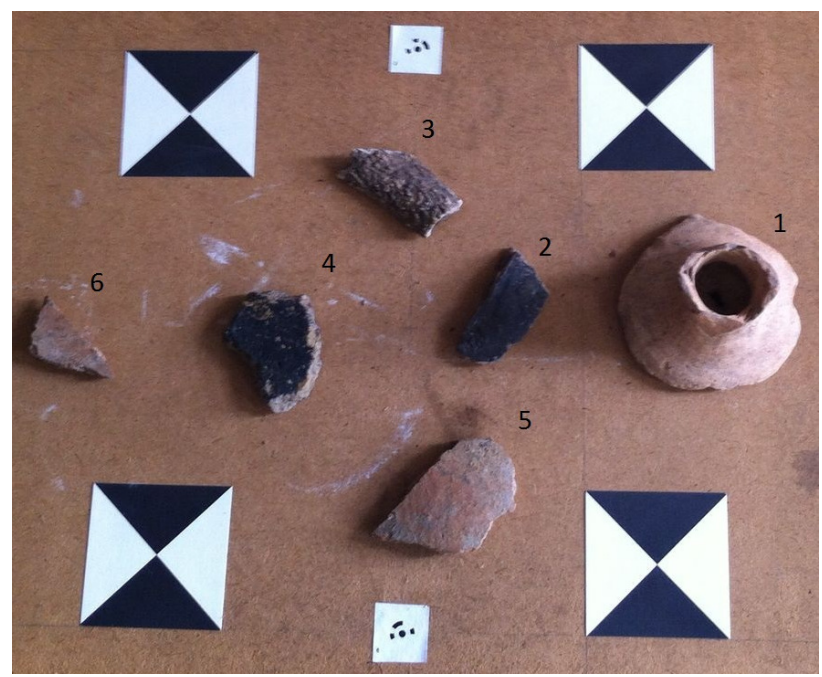

Figure 4. The set of fragments of archaeological artefacts

The most common fragments are made of baked clay, with glaze or without it. That's why we chose all fragments made of this material.

The origin of samples is as follows (numbered as shown in Figure 4):

1. Fragment of Roman ceramic pottery

2. Fragment of prehistory pottery, baked clay with glaze

3. Fragment of ancient bone

4. Fragment of prehistory pottery, baked clay with glaze

5. Fragment of prehistory pottery, baked clay

6. Fragment of prehistory pottery, baked clay

\subsection{Data collection and preprocessing}

All fragments were scanned at spatial resolution $0.5 \mathrm{~mm}$ in both directions. The scanning speed was $1 \mathrm{~mm} / \mathrm{s}$, to reach the same spatial resolution in a direction along the scanning comparing to the direction across the scanning. Computer and dedicated software control the whole scanning process.

After scanning of fragments, the recorded hyperspectral data were preprocessed. First, the number of spectral channels had to be lowered, according to spectral characteristics of ImSpector V9. By division of spectral range $(470 \mathrm{~nm})$ by spectral resolution $(7 \mathrm{~nm})$, it is obvious that ImSpector V9 can separate maximum 67 spectral channels. After it, the value of incident light is substracted from every pixel value of the image, in the same channel. So, the reflexivity of the surface is free from the impact of spectral characteristics of incident light. At the end of the preprocessing phase, all images from the same scene are collected to one hyperspectral image cube.

\subsection{Data analysis}

The data analysis of hyperspectral cube was done by using free software "Hypercube" (Delbosque, 2016)

To check, if proposed method has the potential to find the same fragments. First, we took per 3 samples from the fragments, that reside to the same artefact, according to the analysis made by archaeologists (Figure 5). 


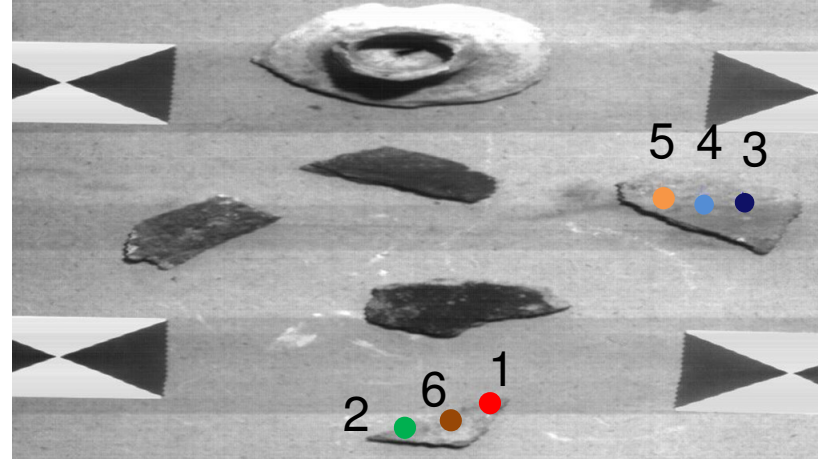

Figure 5. The hyperspectral scene of fragments of archaeological artefacts. The positions used to produce spectral plot are marked by coloured circle and numbered.

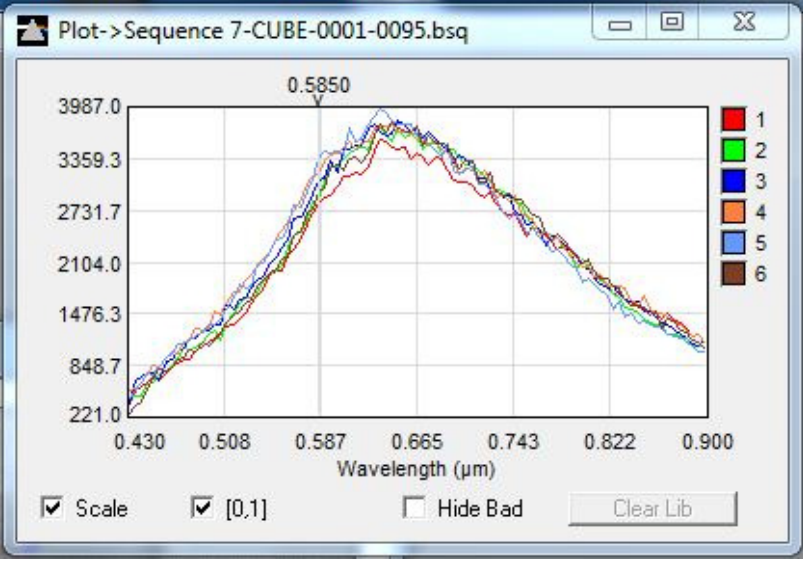

Figure 6. The spectral plot of samples taken from the different places and different fragments that reside to the same artefact.

The number on spectral plot corresponds to the number of sample taken (Figure 5).

Figure 6 shows the very high level of correspondence between all six spectral samples.

Next experiment was done by taking samples from all fragments.

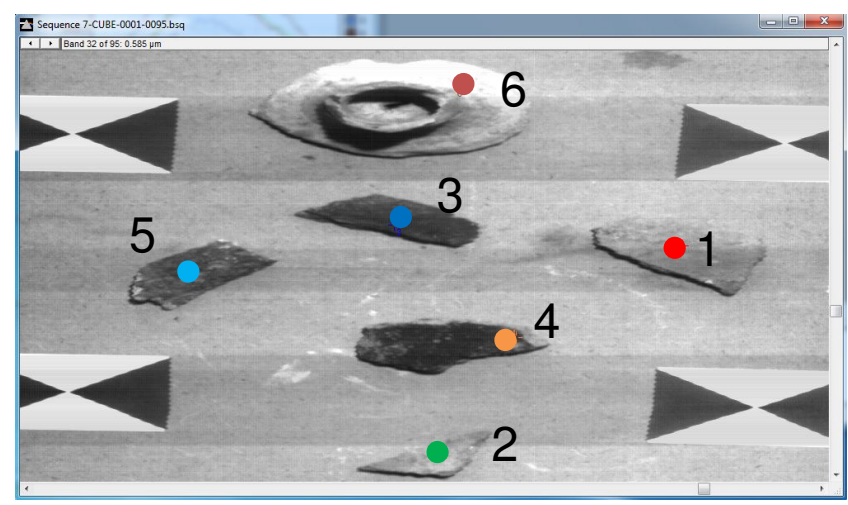

Figure 7. The hyperspectral scene of fragments. All samples were selected for the hyperspectral plot and numbered.

After analysing the hyperspectral plots of different samples (Figure 8), it's clear visible that the plots of fragments numerated 1 and 2, as well as fragments 3 and 4, have a very similar spectral characteristic, almost during the whole visible and NIR spectrum. The best separation of different materials we can reach, if we use the spectral range from 580-680nm.

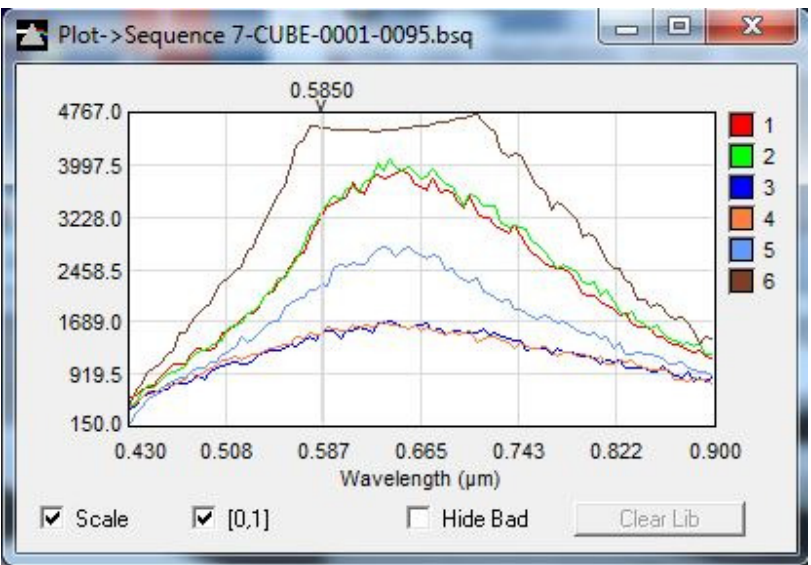

Figure 8. Spectral plot of samples taken from different fragments. The number on spectral plot corresponds to the number written next to sample.

\section{CONCLUSION AND FURTHER WORK}

The presented work shows that the use of hyperspectral scanning technology has a big and real potential in the classification of fragments of archaeological artefacts, with the goal to detect and select those, which originate from the same artefact. The scanning equipment can be build at a reasonable price. It remains to explore and find the best appropriate classification algorithm, which will be able to select the fragments of the same artefacts on the fully automatic way, by the high level of reliability. We started such investigations and the results we will present in our next paper.

Besides detection of fragments of the same artefact, the hyperspectral scanning gives the inevitable information about the spectral characteristic of artefact's surface. It can extend present documentation about archaeological artefact by completely new dimension, the spectral one.

\section{REFERENCES}

Bajić, M., Gold, H., Pračić, Ž., Vuletić,D., 2004. Airborne Sampling of the Reflectivity by the Hyper Spectral Line Scanner in a Visible and Near-Infrared Wavelengths, $24^{\text {th }}$ Symposium of EARSeL, the European Association of Remote Sensing Laboratories, Dubrovnik

Delbosque, B., 2016. Hypercube Software, http://www.erdc.usace.army.mil/Media/FactSheets/FactSheetArticleView/tabi d/9254/Article/610433/hypercube.aspx (05. Apr. 2016)

Lawrence, K.C., Park, B., Windham, W.R., Mao, C., 2003. Calibration of push-broom hyperspectral imaging system for agricultural inspection, Transactions of the ASAE. Vol. 46(2), pp. 513-521

Miljković, V., Gajski, D., 2011. Multispectral Close-Range Facilities Survayes, Professional Practice and Education in Geodesy and Related Fields, University of Belgrade, Faculty of Civil Engineering, pp. 83-88 